\title{
Corela
}

Cognition, représentation, langage

17-1 | 2019

Vol. $17, \mathrm{n}^{\circ} 1$

\section{Impacts du ratage de cible sur la production des voyelles nasales françaises par les apprenants iraniens}

Mohamd Hossein Otroshi, Kamyar Abdoltajedini et Marzieh Mehrabi

\section{(2) OpenEdition}

Édition électronique

URL : http://journals.openedition.org/corela/8239

DOI : $10.4000 /$ corela.8239

ISSN : 1638-573X

Éditeur

Cercle linguistique du Centre et de l'Ouest - CerLICO

Référence électronique

Mohamd Hossein Otroshi, Kamyar Abdoltajedini et Marzieh Mehrabi, «Impacts du ratage de cible sur la production des voyelles nasales françaises par les apprenants iraniens », Corela [En ligne], 17-1 | 2019, mis en ligne le 24 juin 2019, consulté le 27 juin 2019. URL : http:// journals.openedition.org/corela/8239; DOI : 10.4000/corela.8239

Ce document a été généré automatiquement le 27 juin 2019.

\section{(c) (i) (3) (2)}

Corela - cognition, représentation, langage est mis à disposition selon les termes de la licence Creative Commons Attribution - Pas d'Utilisation Commerciale - Partage dans les Mêmes Conditions 4.0 International. 


\title{
Impacts du ratage de cible sur la production des voyelles nasales françaises par les apprenants iraniens
}

\author{
Mohamd Hossein Otroshi, Kamyar Abdoltajedini et Marzieh Mehrabi
}

\section{Introduction}

1 Dans la plupart des systèmes phonétiques, dont le persan, l'anglais et l'arabe, existent des consonnes nasales, mais certains systèmes comme le français, le portugais et le polonais possèdent, en sus de ces dernières, des voyelles nasales. Ces voyelles nasales caractérisant le système phonétique français font l'objet des méthodes d'enseignement et des cours de français langue étrangère (FLE). En effet, la prononciation, bonne ou moins bonne, affecte la communication en langue étrangère. Compte tenu de l'importance de la prononciation, le cadre européen commun de référence (CECR) accorde dans sa grille d'évaluation une place privilégiée à la maitrise du système phonémique. Puisque les voyelles nasales ne caractérisent ni le persan ${ }^{2}$ ni la langue maternelle de la plupart des apprenants iraniens, les enseignants iraniens se verraient souvent confrontés à la question d'en enseigner la prononciation, d'en identifier les occurrences erronées et d'en corriger les occurrences fautives. Dans le système français, les voyelles nasalisées se distinguent des voyelles nasales qui sont phonétiquement différentes et qui entrainent souvent une signification différente, par exemple «jeun [3œ̃] » vs. «jeune [3œn]». Selon des recherche faites, les caractéristiques phonétiques et acoustiques des voyelles nasales du français ne sont pas dues à l'abaissement forcé de l'uvule lors de la production des voyelles orales (correspondantes) (Hansen 1998, Carignan 2012, Delvaux 2012, Montagu 2007, Vaissière 2011, Meunier 2015). Delvaux (2002), Zerling (1990) et Abry et Veldeman-Abry (2007) inventorient les caractéristiques articulatoires des trois voyelles nasales françaises de la façon suivante: la voyelle nasale $[\tilde{\varepsilon}]$ est une articulation (production) centrale non- 
labiale. La voyelle nasale [õ] est plus fermée, plus labiale par rapport à la voyelle nasale [ã̃]. La fréquence et les mesures des formants nous fournissent toujours des indices sur les cavités (bucco-nasale) constituant les formants. Ainsi l'abaissement de la hauteur de la langue ou l'abaissement de celle de la mâchoire inférieure augmente rapidement les mesures du formant 1. La position de la langue sur l'axe postérieur-antérieur et la forme des lèvres, lorsque la langue se positionne dans la partie postérieure de la cavité buccale, augmente les mesures du formant 2. "Le formant 1 et 2 des voyelles nasales sont plus proches l'un de l'autre par rapport à ceux des voyelles orales ». (Dupont et Lejeune 2010 : 9)

2 Meunier (2015:23) voit à l'origine de la formation « des formants nasals de 500 hertz ", entrainant la modulation de la fréquence du «formant 2 », le passage d'une certaine quantité d'air par le nez qui fait résonner les cavités nasales.

3 Le formant 3 est sensible à la longueur de la cavité antérieure lorsque la langue se retire vers la partie antérieure de la bouche et il joue un rôle important dans la caractérisation des voyelles labiales antérieures (Vaissière, 2007). Takemoto et al. (2006 cité par Marchal 2009 : 91) postulent que « le formant 4 se forme dans la cavité supra-laryngienne ».

4 En règle générale, étant donné les six règles décrites par Pickett (1999 : 38-43), la quantité des formants 1 et 2 des voyelles ne se modifie pas considérablement et montre beaucoup de stabilité sur le spectrogramme quand elles se produisent isolément et hors du cotexte lexical ou phrastique. Ce fait est plus ou moins valable pour les voyelles nasales françaises. La figure 1 montre le spectrogramme et les formants 1 et 2 des trois voyelles

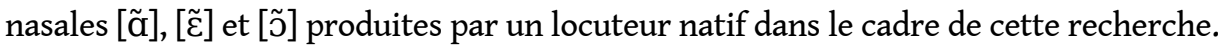

Figure 1. Formants 1 et 2 des trois voyelles nasales françaises produites en isolation (hors contexte lexical ou phrastique).

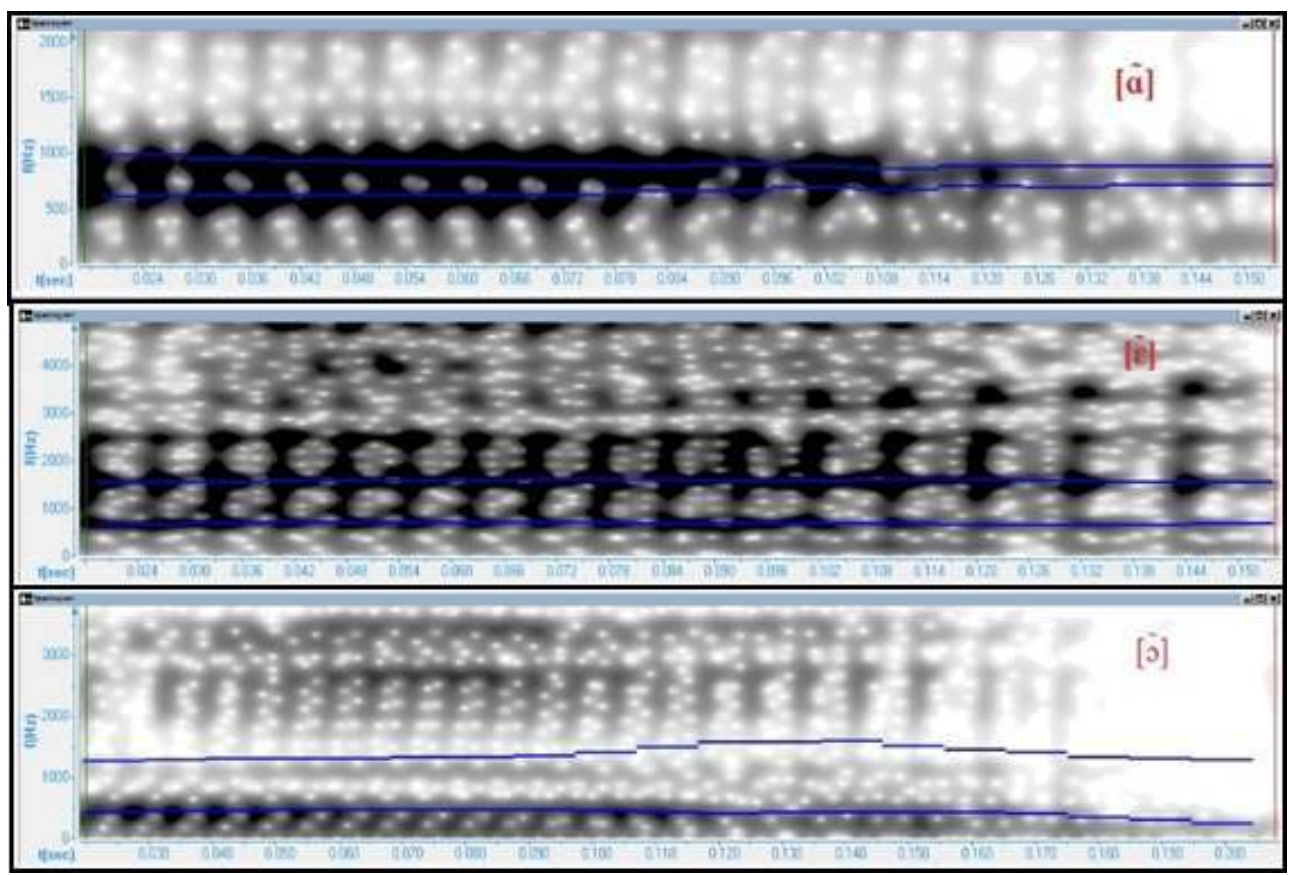

5 Lorsque les voyelles (orales et nasales) se trouvent dans le contexte des sons constituant la chaine parlée, les mesures de leurs formants subissent des modifications acoustiques. Le contexte des sons de la chaine parlée ou de la langue comprend deux types de données : les données langagières émanant de la vertu « phonologique du son » qui sont 
identiques chez tous les locuteurs d'une langue donnée et les données phonétiques qui varient en fonction des caractéristiques individuelles et sociales de chaque locuteur. Ainsi, d'une part, les caractéristiques individuelles et phonétiques influencent les formants (Van Hout 2004 cité par Bijankhan 2013 : 148) et d'autre part, dans le contexte phonétique, la consonne affecte les formants de la voyelle et elle l'éloigne de son propre « hyperespace $»^{3}$.

Dans la présente étude, nous nous penchons d'abord sur l'effet du contexte phonétique sur les formants articulatoires des voyelles nasales françaises, ensuite sur la qualité d'une bonne prononciation (production) et finalement, sur l'identification des écarts figurant dans les productions des locuteurs étudiés.

\section{Historique}

7 Les études scientifiques menées sur les voyelles nasales françaises à l'aide des dispositifs technologiques comme la radiographie ont débuté en 1930 grâce aux travaux de Chlumsky et al. Pendant les décennies ultérieures, des chercheurs comme Delattre (1965), Bothorel et al. (1986), Delvaux (2002), Huffman et Krakow (1993), Montagu (2007), Carignan (2013) et Meunier (2015) se sont servi d'approches telles que la cinéradiographie, l'IRM et l'articulographie électromagnétique pour étudier avec plus de précision les voyelles nasales. La position du phonème nasal dans la syllabe ainsi que dans le mot, le contexte phonétique et la structure suprasegmentale, disent Cléments et al. (2015), sont des facteurs qui s'impliquent entre autres dans la prononciation des voyelles nasales. Étant donné l'existence des pics spectraux provenant des cavités buccale et nasale et des anti-formants, l'étude acoustique et spectrale des voyelles nasales françaises semble compliquée car d'une part, l'indice (le signe) acoustique nasal n'est pas le même pour toutes les voyelles et ne dépend que de la caractéristique vocalique (phonétique) de chaque voyelle et d'autre part, comme nous l'avons déjà dit, l'effet du contexte phonétique empêche les formants d'atteindre leur propre hyperespace. Donc, cela peut engendrer des écarts de prononciation chez des apprenants de FLE.

8 La conception des méthodes d'enseignement et le besoin croissant des enseignants ont poussé, en didactique du français, des chercheurs comme Bilières, Vaissière, Meunier (2015), Abry et Veldeman Abry (2007), Charliac et Motron (2014), Lauret (2007) et Martinie et Wachs (2006) à mener des études sur l'enseignement de la prononciation, l'identification des erreurs concernées et leur correction. Les diverses méthodes d'enseignement du français consacrent une bonne partie à la découverte des structures phonétiques du français. Cependant, dans une classe de FLE, l'enseignant aurait besoin d'une ou de plusieurs approches efficace(s) et rapide(s) pour pouvoir aider ses apprenants à corriger leurs erreurs de prononciation, en fonction des caractéristiques de leur langue maternelle et celles de la langue cible.

Caractérisées par la nasalité, les voyelles nasales françaises sont des structures phonétiques dont la production pourrait poser des difficultés aux apprenants de diverses langues. Dernièrement, l'étude de ces voyelles nasales a intéressé beaucoup de chercheurs en linguistique et en didactique des langues qui ont essayé, à l'aide de la technologie moderne, de les étudier hors de la chaine parlée. Du point de vue phonétique, l'effet des consonnes sur les voyelles est étudié par House et Stevens (1956), Lindblom (1963), Rochette et Gregoire (1983), Rochette et Simard (1985), Hillenbrand et al. (2001) et dans le contexte iranien, Sheikh Sang Tajan et Bijankhan (2010). Pourtant, l'effet du 
contexte phonétique sur les formants et en général l'imbrication phonétique et ses conséquences dans l'occurrence d'erreurs de prononciation chez les apprenants, ont fait moins l'objet d'études scientifiques.

\section{Collecte des données (corpus) et méthodologie d'analyse}

Lors du deuxième semestre de l'année universitaire 2016-2017 nous avons fait une évaluation standard de la prononciation des voyelles nasales du français chez 4 étudiants de première année (3 filles et 1 garçon) d'un âge moyen de 19 ans. Les locuteurs étudiés n'avaient pas de problèmes particuliers au niveau phonatoire. Dans un premier temps, nous avons demandé aux informateurs d'écouter puis répéter individuellement 3 fois deux phrases (table 1) produites par un locuteur natif comme «stimulus et exemplaire phonétique ». Ensuite, nous avons enregistré, à l'aide du logiciel Praat version 6.0.30, les phrases reproduites par les participants. Dans un deuxième temps, nous avons analysé, grâce aux logiciels d'analyse Praat et Speech analyzer, les productions enregistrées de chaque informateur. Pour faire des analyses statistiques, nous nous sommes servis du logiciel Excel 2016. Afin de mesurer l'effet phonétique sur les formants, nous avons mesuré, sur le spectrogramme ainsi que sur la représentation oscillographique de l'onde sonore, la durée des voyelles nasales. Nous avons également segmenté le début et la fin de chaque voyelle. Ensuite nous avons délimité la zone fréquentielle de transition de la consonne à la voyelle nasale et celle de la voyelle nasale à la consonne.

Table 1. Phrases porteuses.

$$
\begin{aligned}
& \text { Il chante une chanson marrante. }
\end{aligned}
$$

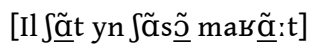

$$
\begin{aligned}
& \text { C'est un roman plein de violence. }
\end{aligned}
$$

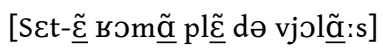

11 Dans la chaine parlée, les sons s'imbriquent dans l'unité de temps, un fait qui modifie la trajectoire des mesures des formants. Dans l'étude et l'analyse de la modification que subirait la trajectoire de fréquence du formant on parle de l'état de transition et de l'état stable. Dans l'état de transition, les mesures du formant n'ont pas une stabilité relative, au contraire dans l'état stable, elles sont relativement stables. Influencée par le point d'émission de la consonne et celui de la voyelle, la trajectoire de la modification de la voyelle peut être stable, descendante, descendante-ascendante, ascendante et ascendante-descendante. Cela veut dire que les mesures des formants des voyelles contiguës aux différentes consonnes varient. Des chercheurs comme Clark et Neary (2001 cité par Bijankhan 2013 : 149) «pensent que l'emplacement de production des consonnes affecte peu le formant 1 des voyelles et que son effet sur le formant 2 des voyelles varie en fonction du type de voyelle et l'emplacement de production de la consonne». 


\section{1 Résultats de l'étude}

\subsection{Voyelle nasale $[\tilde{\varepsilon}]$}

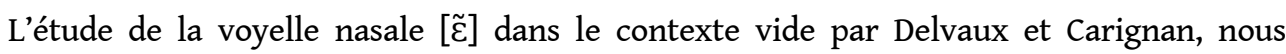
montre la présence de pics spectraux intenses aux alentours de 1500 hertz, et un grand abaissement (l'abaissement des mesures du formant 1 ) ou une augmentation du nombre de pics au-dessous de cette zone fréquentielle, car le formant 1 se divise en deux, sous l'influence d'un anti-formant. Fonagy $(2006: 25)$ postule que « dans la zone fréquentielle 1100 hertz l'espacement du formant 1 et 2 varie et que la zone 1900 hertz est la limite de la formation du formant 2 ». Dans les limites du formant 3, suite à son fort abaissement, se constitue « l'œil nasal ${ }^{4} »$ (figure 2) mais ce dernier disparait si le formant 4 se maintient à la même intensité. L'étude de Carignan (2013) sur les voyelles nasales françaises souligne le fait que la structure orale de la nasalité augmente la fréquence des formants 1 et 2 .

13 La figure et la table suivantes représentent les tracés des formants (lignes rouges) et le spectrogramme de la voyelle nasale [ $\tilde{\varepsilon}]$ produite par le locuteur natif dans les phrases porteuses (table1). Ainsi, puisqu'elle est produite en isolation, elle est débarrassée de l'influence du contexte phonétique (figure et table 2). L'écart moyen entre F2 et F1 pour la

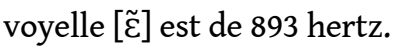

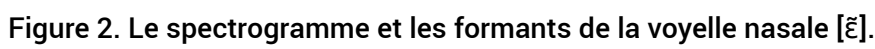

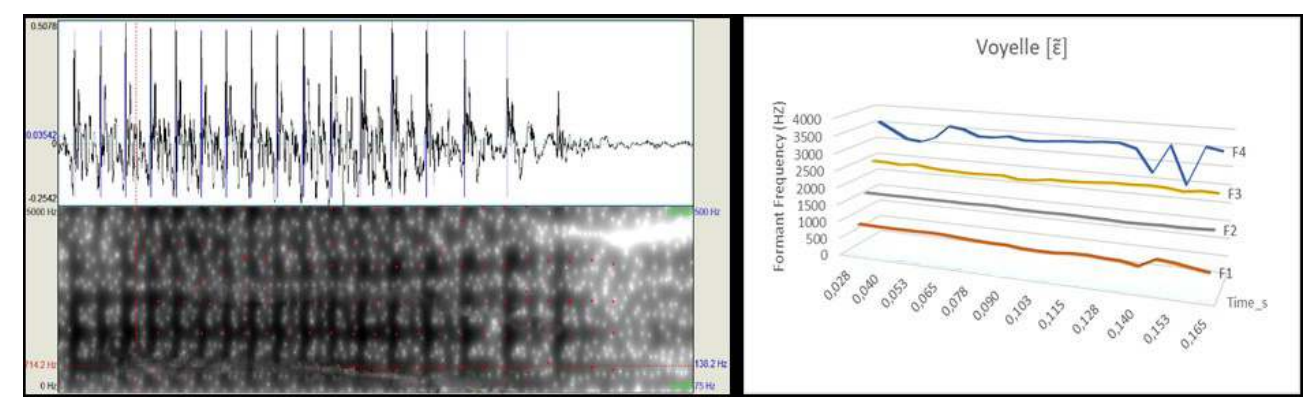

Table 2. Les formants de la voyelle nasale [z̃].

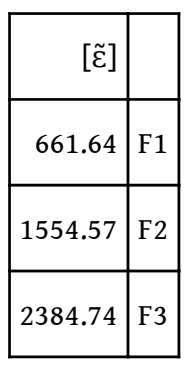

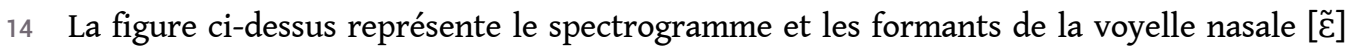
présents dans les mots étudiés et insérés dans les phrases porteuses qui ont été reproduites par le locuteur natif. Dans le contexte phonétique, on voit que les mesures du formant 1 (la hauteur de la langue) et celles du formant 2 (la position de la langue, postériorité ou antériorité) de cette voyelle nasale correspondent à un ratage de cible 
(undershoot), un fort abaissement des mesures (table 3), et que les pics spectraux se situent aux alentours de $1500 \mathrm{~Hz}$. Malgré l'effet du contexte phonétique sur les formants 1 et 2 , la présence des pics montre la présence de l'anti-formant. Influencé par la consonne présente dans les mots porteurs, le formant 3 (forme des lèvres) subit une augmentation relative d'où la constitution de l'œil nasal dans ces limites (figure 3). Cela signifie que dans le contexte, la voyelle $[\tilde{\varepsilon}]$ est influencée par l'imbrication phonétique et malgré l'abaissement des mesures de ses formants 1 et 2 et son recul de "l'hyperespace», elle conserve son formant nasal. Comme le montrent les spectrogrammes ci-après, les caractéristiques acoustiques des sons dans la chaine parlée influencent celles du son qui vient juste après. L'écart moyen des mesures entre $\mathrm{F} 2$ et $\mathrm{F} 1$ pour la voyelle nasale $[\tilde{\varepsilon}]$ présents dans les mots « un » et « plein » est respectivement de 790 et $897 \mathrm{~Hz}$.

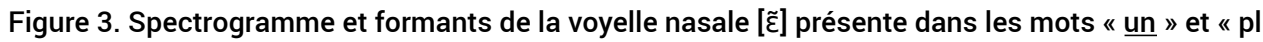
ein ".

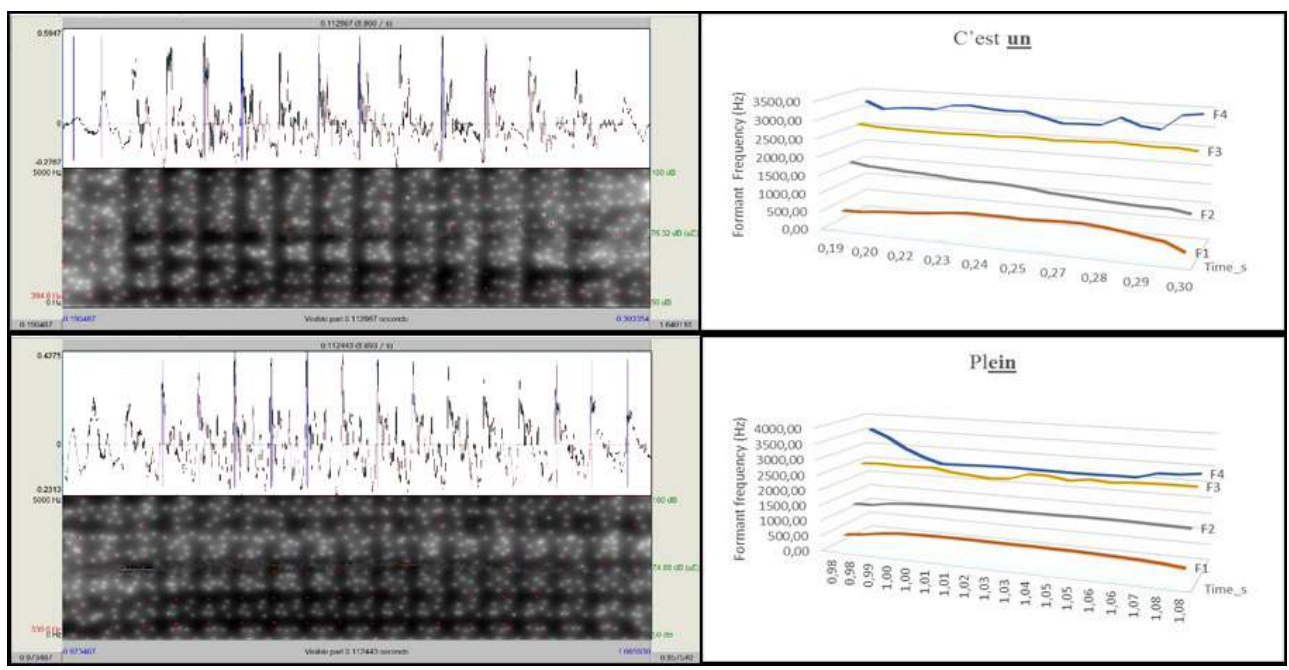

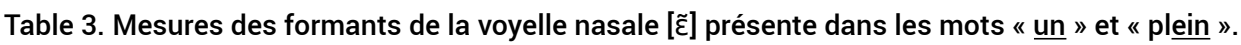

\begin{tabular}{|r|r|l|}
\hline Plein & $\underline{\text { Un }}$ & \\
\hline 502.59 & 522.29 & F1 \\
\hline 1399.59 & 1312.14 & F2 \\
\hline 2457.53 & 2543.56 & F3 \\
\hline
\end{tabular}

La figure suivante représente le spectrogramme et les formants de la voyelle nasale $[\tilde{\varepsilon}]$

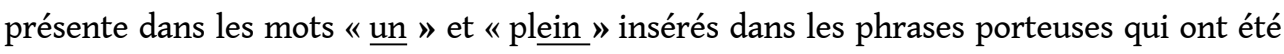
reproduites par l'apprenante 1. L'absence de l'impulsion du larynx pendant certains moments de la reproduction de "un " (figure de gauche) montre la nasalisation de la voyelle, d'où l'apparition d'un écart avec la prononciation standard (phonique). L'abaissement des pics intensifiés entre les deux formants et la durée de production des deux mots montrent une erreur.

Figure 4. Spectrogramme et formants de la voyelle nasale $[\tilde{\varepsilon}]$ présente dans les mots « un » et «plein » produite par le locuteur natif. 


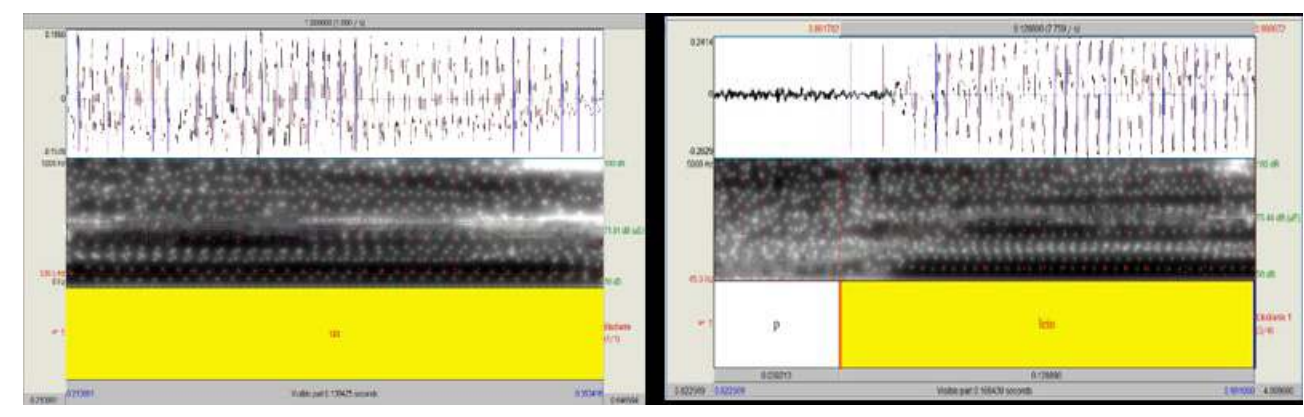

16 En comparant le spectrogramme et les formants de la voyelle nasale [ $\tilde{\varepsilon}]$ produite par le

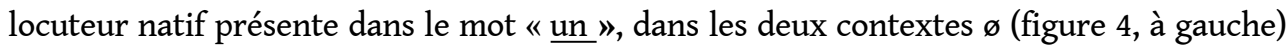
et phonétique (figure 4 , à droite), on voit bien que le chevauchement phonétique entraine la diminution des mesures des formants 1 et 2 ainsi que la diminution de nasalité de la voyelle alors qu'il ne fait pas disparaître le formant nasal. Dans la reproduction phonétique de l'apprenante 1 (figure 5, à droite), tout en constatant l'augmentation de la valeur du formant 2 et la baisse des pics fréquentiels, on voit également que le formant 3 en état de transition ascendant-descendant subit une fluctuation. Ainsi la voyelle nasale reproduite par l'apprenante, subit les modifications qui donnent lieu à l'écart de prononciation.

Figure 5. Comparaison du spectrogramme et des formants de la voyelle nasale [च̃], présente dans le mot " un ", reproduite respectivement de gauche à droite par : le locuteur natif dans le contexte $\emptyset$, dans le contexte phonétique et par l'apprenante 1.

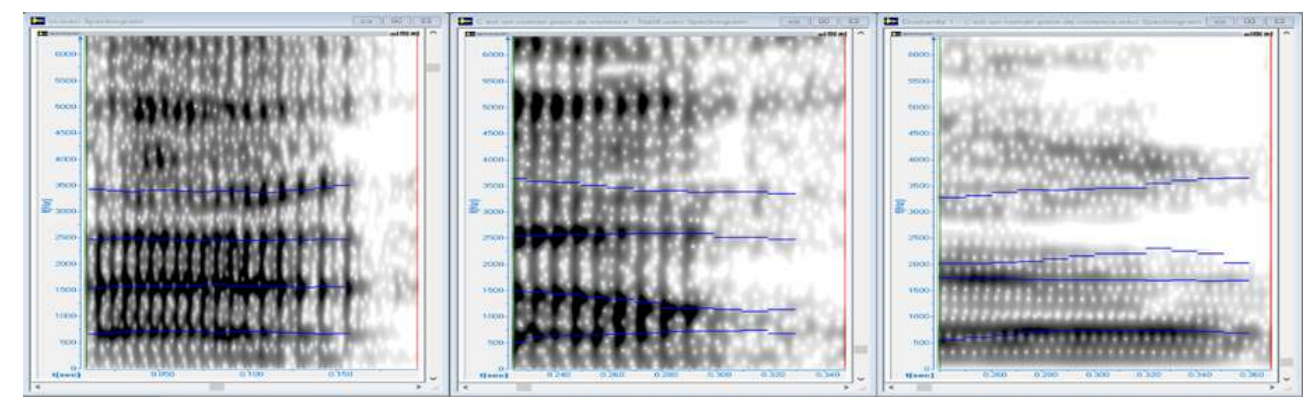

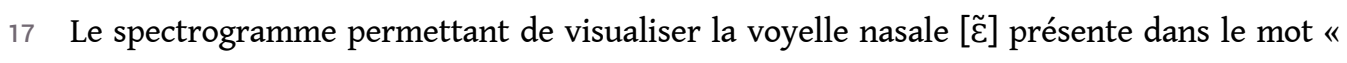
un "reproduite par l'apprenante 2 , démontre la continuité et la densité des pics fréquentiels aux alentours de 500,1500, 3000 et $4000 \mathrm{~Hz}$ ainsi que l'absence de l'œil nasal, à savoir la production d'une voyelle orale au lieu d'une voyelle nasale. Apparemment, les apprenants utilisent une stratégie de production : on produit une voyelle orale dépourvue d'anti-formant au lieu de produire une voyelle nasale. Ce phénomène se répète lors de la production du mot " plein ", aux alentours des plages de fréquence comprises entre 300 à $1000 \mathrm{~Hz}$ et 2500 à $3500 \mathrm{~Hz}$.

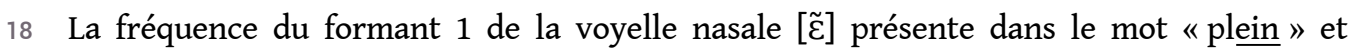
reproduite par l'apprenant 4 , n'a pas subi de diminution des mesures, voire les mesures de son formant $2(1662,2 \mathrm{~Hz})$ ont aussi augmenté. Les deux formants 1 et 2 se maintiennent dans un état stable mais le formant 3 présente une altération descendanteascendante et à certains moments, se rapproche du formant 2 et empêche la formation de l'œil nasal. 


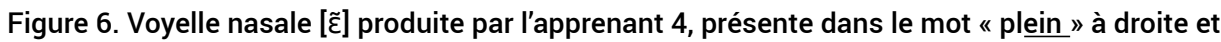
dans le mot « un » à gauche.

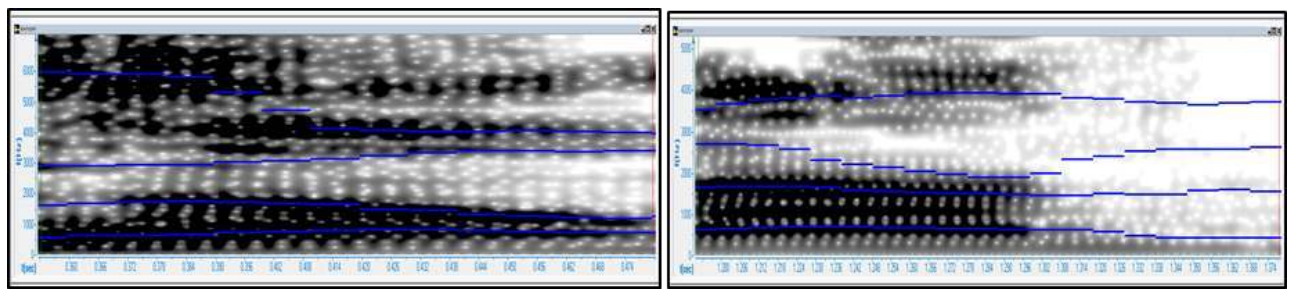

19 Les tables suivantes représentent les mesures des formants 1, 2 et 3 de la voyelle nasale $[\tilde{\varepsilon}]$, présente dans les mots « $\underline{\text { un }}$ " et "plein » et produite par le public étudié. Malgré les efforts des apprenants étudiés pour reproduire correctement la voyelle nasale, la nonformation d'anti-formant et par conséquent, celle de l'œil ou du murmure nasal

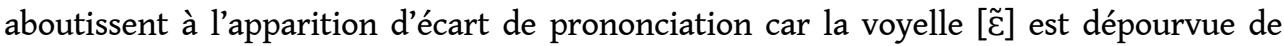
nasalité. Le fait que la voyelle nasale se trouve dans un contexte phonétique et que la consonne affecte les formants, empêche la reproduction appropriée de la voyelle nasale par les apprenants.

Présenté dans l'ordre des apprenants, l'écart moyen des mesures entre F2 et F1 pour la voyelle nasale $[\tilde{\varepsilon}]$ présente dans le mot « un » est de 1225, 697, 1098 et $1069 \mathrm{~Hz}$ et dans le mot « plein » est de 767, 1172, 1034 et $1027 \mathrm{~Hz}$.

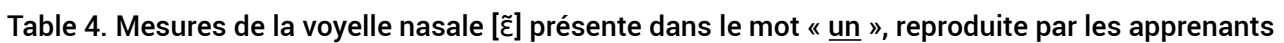
étudiés.

\begin{tabular}{|r|r|r|r|r|}
\hline S4 & S3 & S2 & S1 & \\
\hline 555.7 & 628.5 & 605.8 & 544.3 & F1 \\
\hline 1624.5 & 1726.1 & 1302.1 & 1769.2 & F2 \\
\hline 2899.5 & 2988.9 & 2733.1 & 2013.3 & F3 \\
\hline
\end{tabular}

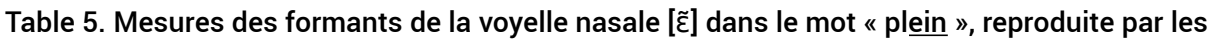
apprenants.

\begin{tabular}{|r|r|r|r|r|}
\hline $\mathrm{S} 4$ & $\mathrm{~S} 3$ & $\mathrm{~S} 2$ & $\mathrm{~S} 1$ & \\
\hline 635.0 & 384.9 & 600.7 & 367.6 & $\mathrm{~F} 1$ \\
\hline 1662.2 & 1418.1 & 1772.9 & 1134.8 & $\mathrm{~F} 2$ \\
\hline 2686.5 & 2788.2 & 2570.4 & 2163.8 & $\mathrm{~F} 3$ \\
\hline
\end{tabular}

\subsection{Voyelle nasale [ã]}

On constate, aux alentours de $1000 \mathrm{~Hz}$ de la voyelle [ũ], la formation d'un pic intense suite à la concentration des formants 1 et 2 , (Vaissière, 2007), ainsi que le maintien de stabilité 
du rapprochement de ceux-ci. Dans ses études, Fonagy (2006: 25) montre que «le formant 2 se situe dans les alentours de $1300 \mathrm{~Hz}$ et que la distance du formant 1 et du formant 2 varie dans les alentours fréquentiels de $650 \mathrm{~Hz}$ ». Dans les alentours fréquentiels de $3500 \mathrm{~Hz}$, se forme un autre pic intense alors que la zone fréquentielle du formant 3 subit une réduction qui donne lieu à la formation de l'œil nasal. L'écart moyen des mesures entre F2 et F1 de la voyelle [ã] est de $666 \mathrm{~Hz}$.

Figure 7. Spectrogramme, formants et LPC de la voyelle nasale [ã] reproduite par le locuteur natif dans le contexte $\emptyset$.

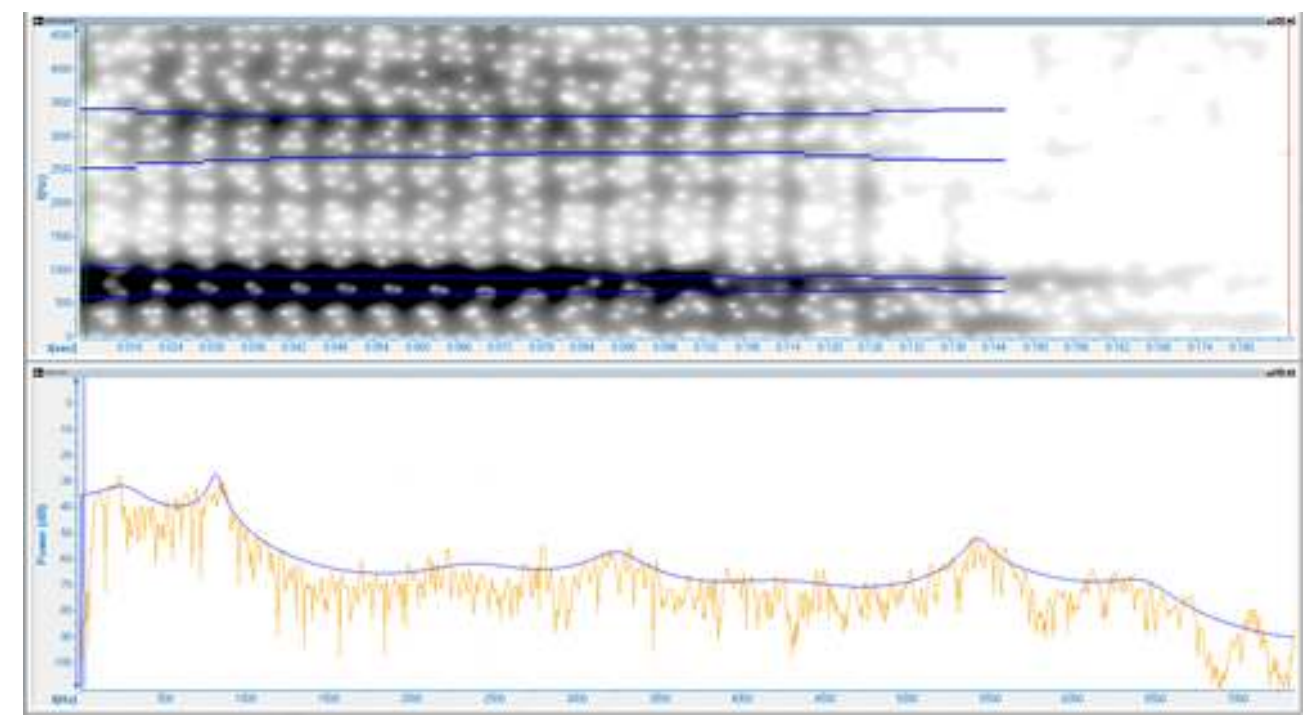

Table 6. Mesures des formants de la voyelle nasale [ã] dans le contexte $\emptyset$.

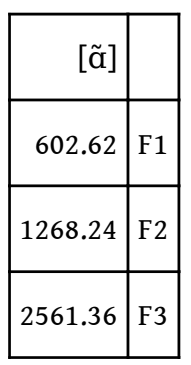

Correspondant à un ratage de cible, les formants 1 et 2 de la voyelle nasale du mot « rom an >», située dans la partie terminale (CVCVn), subissent une diminution des formants sous l'influence de l'imbrication phonétique (figure 8). Les pics spectraux se voient toujours aux alentours de $1000 \mathrm{~Hz}$ alors qu'ils sont fortement abaissés dans les limites de 500 à $750 \mathrm{~Hz}$. La trajectoire de modification des mesures du formant 2 est transitoire et descendante alors qu'elle se stabilise dans la suite de la production. On y voit une diminution de $138 \mathrm{~Hz}$ du formant 3, la décroissance des pics spectraux dans les limites de $3500 \mathrm{~Hz}$ et la formation de l'œil nasal. Influencée par la consonne nasale [m], la voyelle nasale [ã̃] conserve ses vertus nasales malgré la diminution des mesures des formants 1, 2 et 3. Motivé par l'interposition de la voyelle nasale entre deux consonnes (CVCVnC), les formants 1, 2 et 3 de la voyelle nasale [ã], dans le mot "violence ", subissent une baisse de fréquences (table 7), accompagnée d'un décroissement de l'intensité des pics spectraux dans les limites de 1000 et $3500 \mathrm{~Hz}$. Malgré la baisse des fréquences, l'œil nasal se voit 
bien. L'écart moyen des mesures entre F2 et F1 pour la voyelle [ã ] présente dans les mots « roman » et « violence », est respectivement de 526 et $718 \mathrm{~Hz}$.

Figure 8. Spectrogramme et formants de la voyelle nasale [ã] présente dans le mot « roman » au dessus et dans le mot « violence » en dessous.

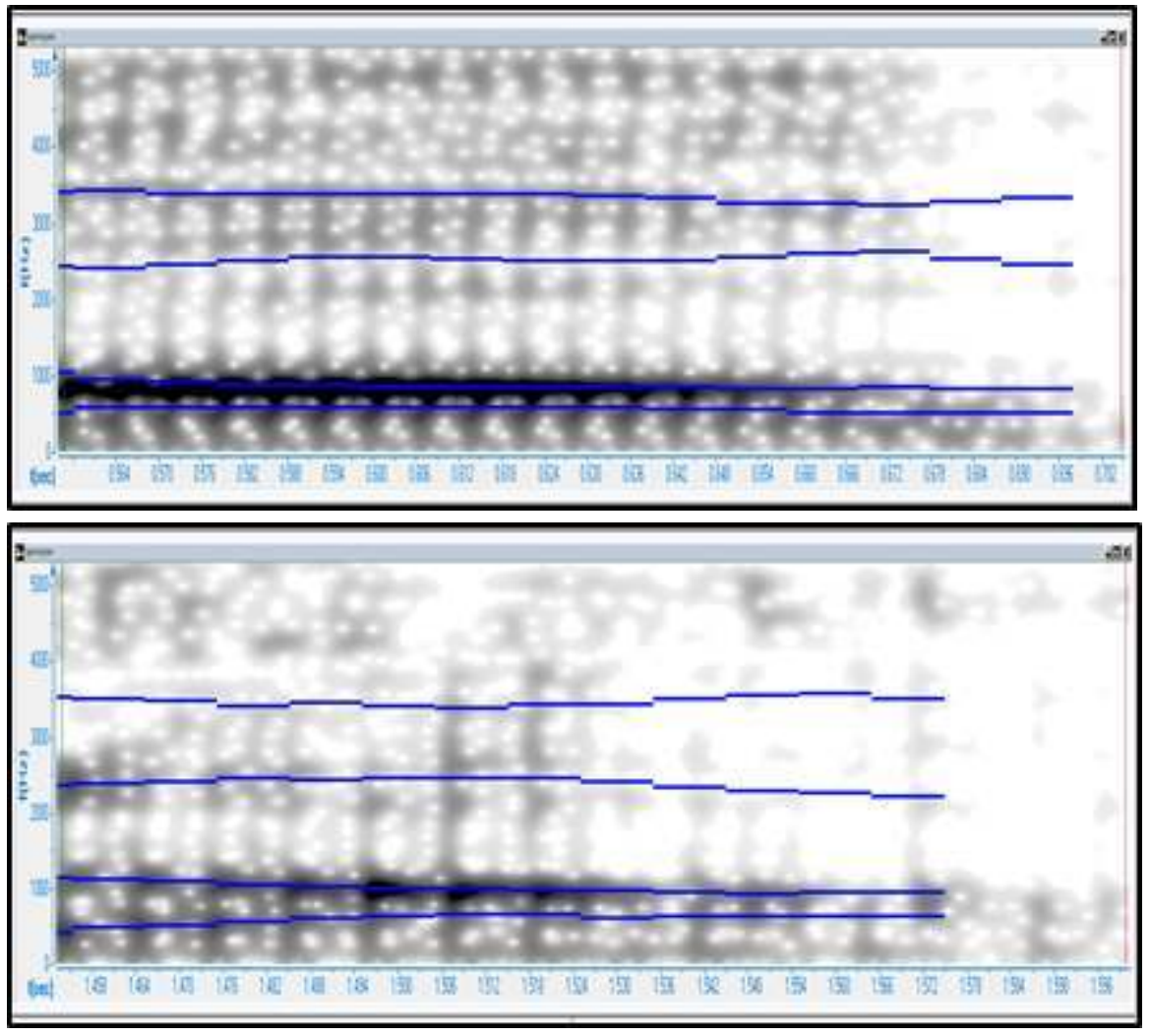

Table 7. Mesures des formants de la voyelle [ã] présente dans les mots « roman » et « violence ».

\begin{tabular}{|r|r|l|}
\hline Violence & Roman & \\
\hline 424.0 & 513.2 & F1 \\
\hline 1142.7 & 1039.0 & F2 \\
\hline 2363.9 & 2423.5 & F3 \\
\hline
\end{tabular}

23 Le spectrogramme et les formants de la voyelle nasale [ãa] reproduite par l'apprenante 1 , présents dans les mots "roman " et "violence " qui sont insérés eux-mêmes dans une phrase porteuse, témoignent d'un écart de prononciation (figure 9). On y voit que le trajet de modification des quantités des formants 1 et 2 , dans le mot « roman ", est instable et que le formant 2 se divise visiblement en deux formants dans la partie terminale. Subissant une forte augmentation fréquentielle, le formant 3, atteint $3656 \mathrm{~Hz}$, s'éloigne ainsi de son propre espace et disparait au milieu du trajet. Dans les limites de 1000 et 3500 $\mathrm{Hz}$ ne se forme aucun pic intense, et on relève le même écart que l'on observe dans le mot «violence ». On peut donc conclure que l'œil nasal ne se forme pas sous l'emprise de la 
modification des formants et de l'emplacement de production des formants, d'où l'apparition d'écart de prononciation.

Figure 9. Spectrogramme et formants de la voyelle nasale [ã] présente dans le mot « roman » à gauche et le mot « violence » à droite.
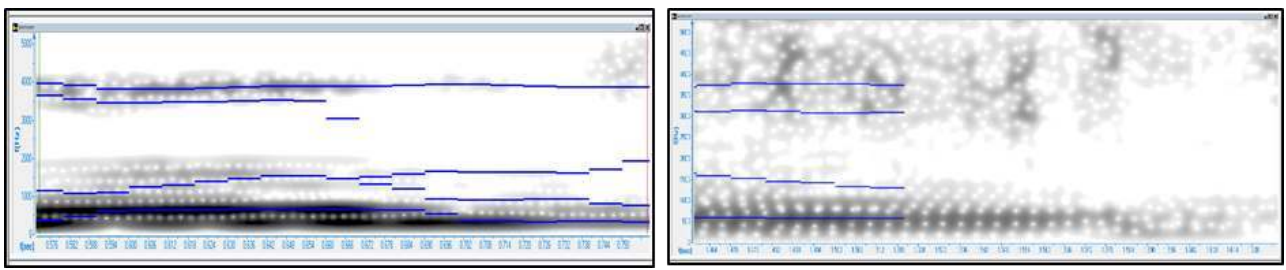

Cet écart de prononciation se voit également dans les reproductions de l'apprenante 2 . Lors de la reproduction qu'elle fait de cette voyelle présente dans le mot "roman ", se forment des pics spectraux intenses dans l'espace des formants 1 et 2 qui caractérisent en effet la production de la voyelle orale. On y voit que l'augmentation fréquentielle du formant 3 entraine la disparition de l'œil nasal et que la croissance d'intensité des pics spectraux fait que le formant 4 se trouve dans les limites de $4000 \mathrm{~Hz}$. Comme le montre la figure suivante, les mesures des formants de cette voyelle nasale reproduite par cette apprenante présentent des écarts. Vaissière (2008 cité par Cléments et al. 2015) postule que la vertu nasale qui commence dans la deuxième partie de la voyelle et qui se prolonge avec un murmure nasal moins fort dans la consonne, permet de percevoir cette qualité phonétique et qu'on ne perçoit pas sa nasalité si le murmure nasal est bref.

Figure 10. Spectrogramme et formants de la voyelle nasale [ã] présente dans les mots « roman » (à gauche) et « violence » (à droite).

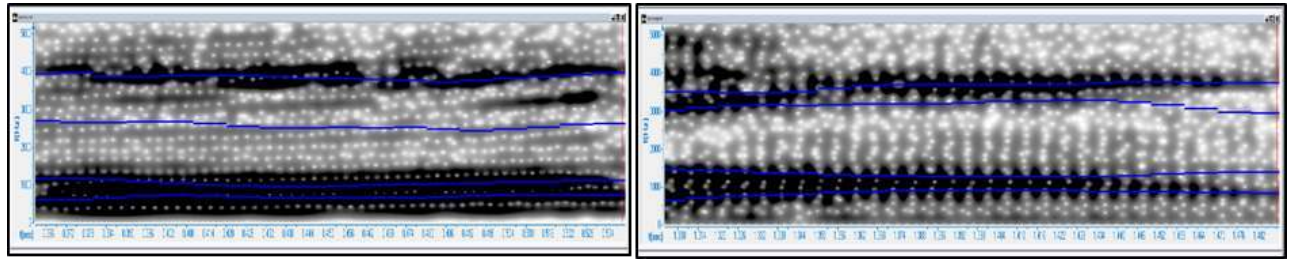

Table 8. Mesures des formants de la voyelle nasale [ã] reproduite par les apprenants étudiés, présente dans le mot « roman ».

\begin{tabular}{|r|r|r|r|r|}
\hline S4 & S3 & S2 & S1 & \\
\hline 674.6 & 631.3 & 601.7 & 357.9 & F1 \\
\hline 915.5 & 1158.9 & 1165.5 & 1131.8 & F2 \\
\hline 2560.8 & 2053.4 & 2705.9 & 3656.0 & F3 \\
\hline
\end{tabular}

Lorsque la consonne nasale précède la voyelle nasale, comme dans le mot "roman ", l'écart est plus évident, car l'apprenant, influencé par la consonne nasale, est amené à produire une voyelle orale. L'écart moyen des mesures de F2 et F1 de la voyelle nasale [ã] présente dans le mot « roman " est respectivement dans l'ordre des apprenants étudiés $774,574,527$ et 241 hertz et pour celle présente dans le mot « violence », de 995, 821, 742, 
892 hertz. Dans la production de cette voyelle nasale, la moyenne des mesures du formant 3 joue un rôle déterminant, dont l'écart par rapport à la norme révèle un écart phonétique (tables 8 et 9 ).

Table 9. Mesures des formants de la voyelle nasale [ã] produite par les participés, présente dans le mot "violence"

\begin{tabular}{|r|r|r|r|r|}
\hline $\mathrm{S} 4$ & $\mathrm{~S} 3$ & $\mathrm{~S} 2$ & $\mathrm{~S} 1$ & \\
\hline 644.6 & 626.2 & 640.2 & 588.8 & $\mathrm{~F} 1$ \\
\hline 1536.5 & 1368.7 & 1461.9 & 1583.0 & $\mathrm{~F} 2$ \\
\hline 2713.0 & 2643.9 & 3017.7 & 3109.2 & $\mathrm{~F} 3$ \\
\hline
\end{tabular}

\subsection{Voyelle nasale [ร̃]}

Dans le spectrogramme ci-après représentant la voyelle nasale [õ], on voit deux pics spectraux faibles dans les limites de $1000 \mathrm{~Hz}$ (Carignan, 2012) et une hausse spectrale dans la zone supérieure à $3500 \mathrm{~Hz}$, ainsi qu'une intensification spectrale. Montagu (2007) et Carignan postulent que le premier et le deuxième pic sont, sous l'influence de la nasalité, toujours faibles. La figure et la table suivantes comprennent le spectrogramme (figure 11) et les mesures des formants (table 10) de la voyelle nasale [0̃] présente dans le contexte vide.

Figure 11. Spectrogramme et formants de la voyelle nasale [ว̃] produite par le locuteur natif dans le contexte vide.

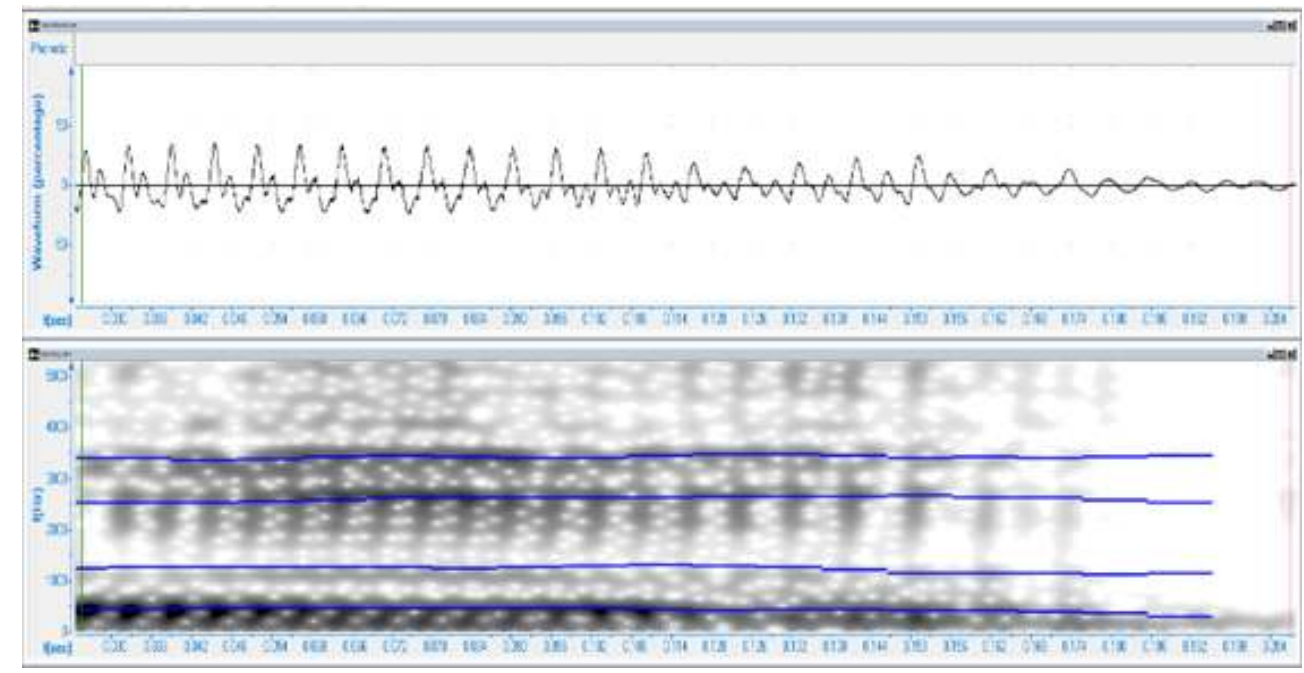

Table 10. Mesures des formants de la voyelle nasale [ว̃] dans le contexte vide.

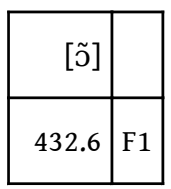




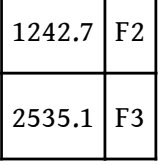

Les formants 1 et 2 , voire 3 de la voyelle nasale [õ], du substantif étudié "chanson ", présente dans le contexte phonétique, subissent une hausse de la valeur des formants (figure 12). Ainsi l'écart moyen entre les mesures de F2 et F1 de la voyelle nasale [õ], présente dans le contexte vide et phonétique, atteint respectivement $810 \mathrm{~Hz}$ et $1167 \mathrm{~Hz}$ et son formant 3 s'approche du formant 4 . Alors que cette augmentation des mesures est en corrélation antinomique avec la formation des pics spectraux intenses dans les limites de 500 et $3500 \mathrm{~Hz}$, elle ne fait pas disparaitre le murmure nasal (table 11).

Figure 12. Spectrogramme et formants de la voyelle nasale [כ̃] présente dans le mot « chanson ».

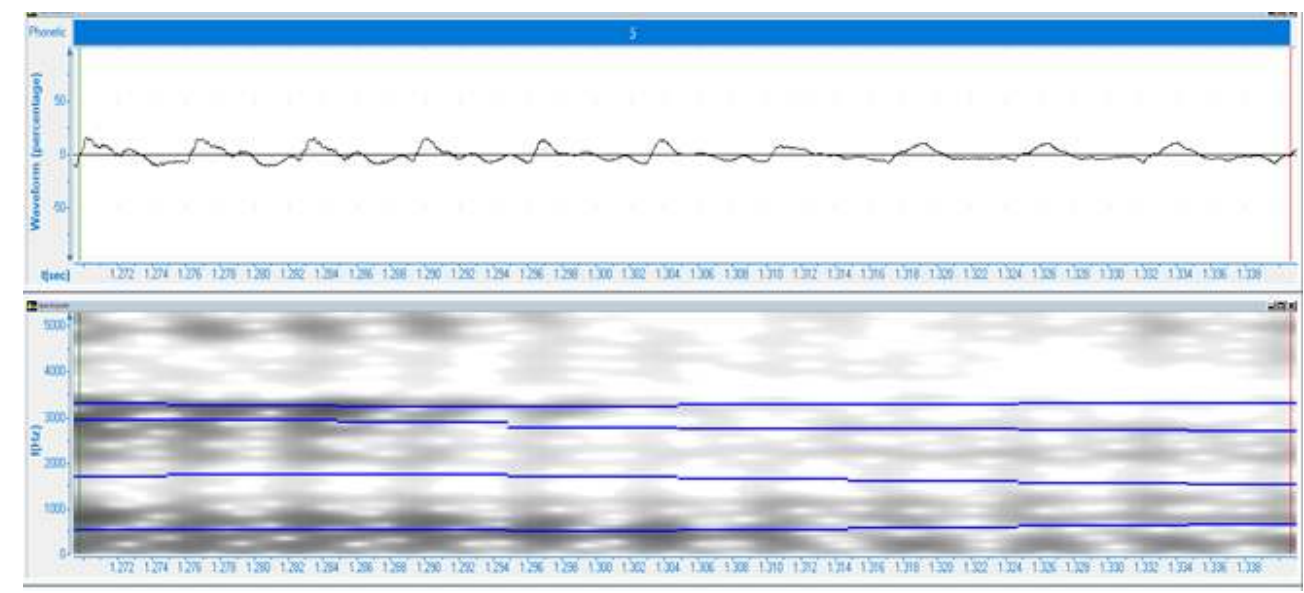

Table 11. Mesures des formants de la voyelle [j̃] présente dans le mot « chanson »

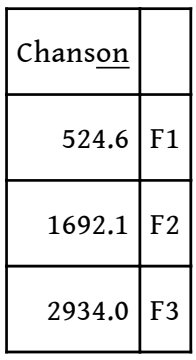

Dans la production phonétique de l'apprenante 1 , on voit que le trajet du formant 2 de la voyelle [õ], présente dans " chanson ", est ascendant-descendant, les mesures du formant 3 s'augmentent et que le trajet de celui-ci est descendant à mi-chemin. Dans la prononciation de cette voyelle, comme on le voit dans la figure ci-après, l'œil ou le murmure nasal n'est pas formé, l'intensité spectrale dans les limites de $3500 \mathrm{~Hz}$ ne se voit pas, à savoir qu'il y a un écart dans la prononciation de l'apprenante. 
Figure 13. Spectrogramme et formants de la voyelle nasale [כ̃] produite par l'apprenante 1, présente dans le mot " chanson ».

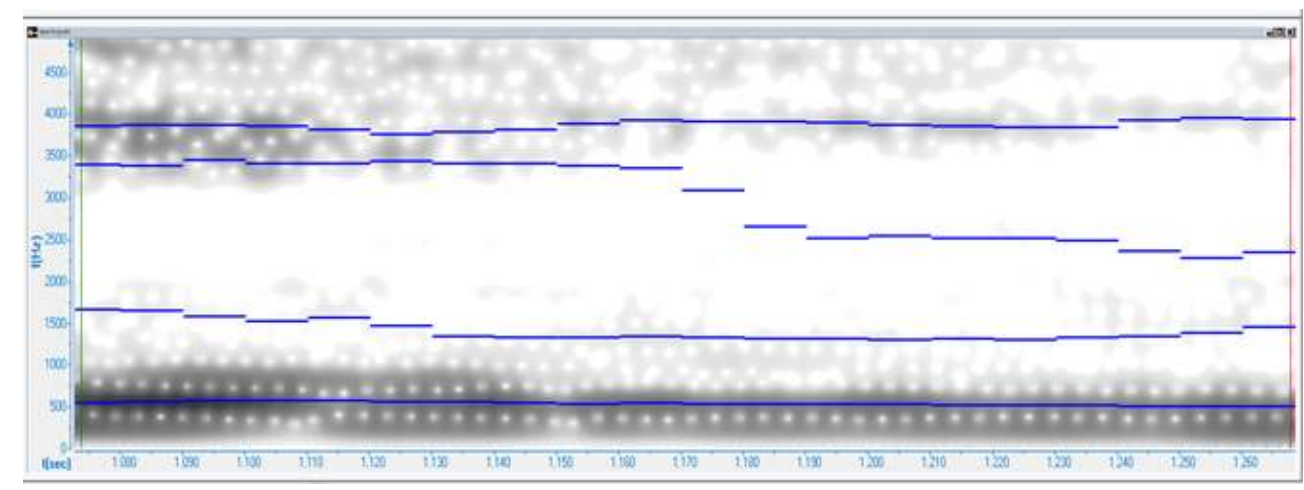

Nous avons constaté chez les autres apprenants que nous avons étudiés, l'apparition d'écart de prononciation lors de la production de la voyelle nasale [õ] présente dans le mot «chanson ». En effet, la haute fréquence du formant 3 dans leur production et l'intensité des pics spectraux dans les limites de 1000 et $4000 \mathrm{~Hz}$ empêchent la formation du murmure nasal (figure 14). L'augmentation des mesures du formant 4 jusqu'à $5934 \mathrm{~Hz}$ chez l'apprenant 4 , nous amène à croire que l'apprenant a encore besoin d'entrainement pour pouvoir contrôler son appareil phonatoire. Il est à noter que la proportion des formants joue un rôle important dans la production correcte des sons. Or, dans un premier temps, la proportion des mesures de F2 moins celles de F1, c'est-à-dire (F2-F1) et dans un deuxième temps celle de F3 moins F2 permettent d'identifier une production correcte (Table 12).

Figure 14. Spectrogramme et formants de la voyelle nasale [כ̃] dans le mot étudié "chanson ", produite respectivement (de gauche à droit) par les apprenantes 2 et 3 .

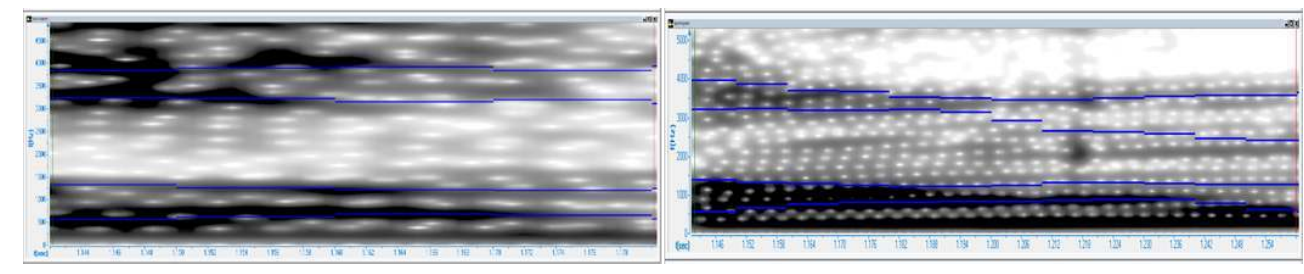

Table 12. Mesures des formants de la voyelle nasale [כ̃] présente dans " chanson ", produite par les apprenants étudiés.

\begin{tabular}{|r|r|r|r|r|}
\hline S4 & S3 & S2 & S1 & \\
\hline 605.0 & 554.7 & 559.7 & 538.2 & F1 \\
\hline 1419.7 & 1386.6 & 1322.8 & 1667.7 & F2 \\
\hline 3261.6 & 3214.9 & 3238.3 & 3392.5 & F3 \\
\hline
\end{tabular}




\section{Conclusion} trois voyelles nasales françaises dans le contexte vide pose problème au public étudié. Corroborés avec ceux-ci, les résultats de la présente étude montrent ainsi que sous l'effet du ratage de cible et celui des caractéristiques nasales (murmure et/ou œil nasal), les voyelles nasales produites par les 4 apprenants sont également affectées d'écart de prononciation dans le contexte phonétique. Ceci provient de la disproportion des mesures des formants 1, 2 et 3 qui explique ainsi l'écart au niveau du timbre des voyelles. La proportion des formants 1,2 et 3 des voyelles nasales produites par les apprenants montre un écart important par rapport à celle des mêmes voyelles produites par les 3 locuteurs natifs.

L'analyse acoustique des voyelles nasales dans les mots étudiés relève que la position de la syllabe où se trouve la voyelle nasale influence le processus de prononciation: A. L'écart phonétique lié à la production des voyelles nasales présentent dans les syllabes initiales et/ou médianes est plus apparent, et la conversion des voyelles nasales en consonne se fait plus souvent dans les mots dont la structure syllabique est plutôt fermée (VnC). Au contraire, nous n'avons pas constaté autant de conversion en consonne dans les mots dont la structure syllabique est ouverte (CVn), un constat qui est corroboré par les résultats de l'étude de Detey et al. (2010). L'apprenant utilise la stratégie de découpage (unpacking, c'est-à-dire le processus par lequel une voyelle nasale se transforme en séquence Voyelle orale + Consonne nasale) des sons et produit deux sons quand il s'agit de produire une voyelle nasale. Et sous l'effet du fonctionnement syllabique persan, la consonne nasale $/ \mathrm{n} /$ rand semi-nasale la voyelle qui la précède. Par exemple : Plein / plan/* ou /pelan/*; Bon /bon/*; Violence /violans/*. B. Il ressort de l'analyse des données qu'il y a une forte corrélation entre l'écart phonétique touchant la voyelle nasale et la coexistence de deux voyelles nasales dans le même mot, comme dans le mot « chans on ". Cela pourrait s'expliquer par le fait qu'en persan même lorsqu'il y a deux consonnes successives, elles ont tendance à être séparées et à s'attacher chacune à la voyelle proche, par exemple, kardak [kār-dak].

BIBLIOGRAPHIE

ABRY D. et VELDEMAN-ABRY, J. (2007), La phonétique : audition, prononciation, correction, Paris, CLE. BIJANKHAN M. (2013), Phonetic system of the Persian language, Tehran, SAMT.

BOERSMA P. et WEENINK D. (2017), Praat : doing phonetics by computer (version 6.0.30), Amsterdam, University of Amsterdam.

CARIGNAN C. (2012), Quand nasal est plus que nasal : L'articulation orale des voyelles nasales en français, Actes de la conférence conjointe JEP-TALN-RECITAL, vol. 1 : JEP, p. 747-754

Corela, $17-1$ | 2019 
CARIGNAN C., SHOSTED R., FU M., LIANG Z. P., \& SUTTON B. (2013), The role of the pharynx and tongue in enhancement of vowel nasalization : A real-time MRI investigation of French nasal vowels, In Proceedings of the Annual Conference of the International Speech Communication Association, INTERSPEECH (pp. 3042-3046), International Speech and Communication Association.

CHARLIAC L. et MOTRON A.-C. (2014), Phonétique progressive du français, Deuxième édition, Paris, CLE.

CLEMENTS G.N. \& al. (2015), The feature [nasal]. In Rialland A., Ridouane, R. \& Van der Hulst, H. Features in phonology and phonetics : posthumous writings by Nick Clements and coauthors ( $\mathrm{p}$. 195-21), Berlin, De Gruyter.

CONSEIL DE L'EUROPE (2005), Cadre européen commun de référence pour les langues, Division des politiques linguistiques Strasbourg, Editions Didier.

DETEY S., RACINE I., KAWAGUCHI Y., ZAY F., BUEHLER N. \& SCHWAB S. (2010), Evaluation des voyelles nasales en français L2 en production : de la nécessité d'un corpus multitâches. 2 ème Congrès Mondial de Linguistique Française. In F. Neveu, V. Muni-Toké, J. Durand, T. Klingler, L. Mondada \& S. Prévost (eds.), Actes de CMLF'10, Paris, ILF, p. 1289-1301.

DUPONT M. et LEJEUNE B. (2010), Rééducation de la boucle audio-phonatoire : Chez les adultes sourds porteurs d'un implant cochléaire, Issy-les-Moulineaux, Elsevier Masson.

FONAGY I. (2006), Dynamique et changement, Louvain-Paris, Peetres.

HANSEN A.-B. (1998), Les voyelles nasales du français parisien modern, Copenhagen, Museum Tusculanum press.

HILLENBRAND J.-M. \& al. (2001), Effects of consonant environment on vowel formant patterns, Journal of the Acoustical Society of America, 109(2), p. 748-763.

HUFFMAN M. K. \& KRAKOW R. A. (1993), Phonetics and Phonology: Nasals, Nasalization, and the Velum , London, Academic press.

LINDBLOM, B. (1963), Spectrographic study of vowel recognition, Journal of the Acoustical Society of America, 35(2), p. 1773-1781.

LAURET B. (2007), Enseigner la prononciation du français : questions et outils, Paris, Hachette.

MARCHAL A. (2009), From Speech Physiology to Linguistic Phonetics, London, ISTE.

MARTINIE B. \& WACHS S. (2006), Phonétique en dialogue, Paris, CLE.

MEUNIER C. (2015), Petit Guide pratique de la Phonétique corrective du FLE, Marseille, Edition du FLE Marseille.

MONTAGU J. (2007), Analyse acoustique et perceptive des voyelles nasales et nasalisées du français parisien, Thèse de Doctorat, Paris-3.

OTROSHI M.H. (2017), The Use of Acoustic Phonetics in Identifying Phonetic Errors : French Nasal Vowels, Language Related Research, vol. 8, No.3 (Tome 38), p. 187-216.

PICKETT J. M. (1999), The Acoustics of Speech Communication : Fundamentals, Speech Perception, Theory, and Technology, London, Allyn \& Bacon.

ROCHETTE C.-E. et GREGOIRE L. (1983), Contribution à l'étude des coarticulations de consonnes occlusives et de voyelles en français, Phonétique combinatoire I, B-120, Québec, CIRB.

ROCHETTE C.-E. et SIMARD C. (1985), Étude des séquences de type consonne constrictive plus voyelle en français, à l'aide de la radiocinématographie et de l'oscillographie, Phonétique combinatoire II, B-148, Québec, CIRB. 
SHEYKH SANGTAJAN SH. \& BIJANKHAN M. (2010), The study of vowel reduction in Persian spontaneous speech, Linguistics Research, Volume 2, Issue 2, p. 35-48.

VAISSIERE J. (2007), Experimental approaches to phonology, Oxford, Oxford University Press.

VAISSIERE J. (2006), La phonétique. Que sais-je ?, Paris, Presses universitaires de France.

\section{NOTES}

1. Ratage de cible

2. Langue officielle du pays

3. Espace vocalique d'une langue donnée où se produit toute voyelle dans sa forme idéale.

4. Quand les formants d'une voyelle nasale constituent une forme ressemblant à un œil, on parle d'œil nasal.

\section{RÉSUMÉS}

La présente recherche s'intéresse à l'impact du contexte phonétique sur les formants 1, 2 et 3 des voyelles nasales françaises. La chaine parlée en français est constituée de la succession des voyelles orales et nasales influencées par le contexte consonantique. En nous appuyant, dans cette étude, sur le modèle de Stevens et House, dit " production undershoot model » ${ }^{1}$, nous avons abordé plus particulièrement l'effet du contexte phonétique sur les trois voyelles nasales françaises afin de vérifier l'hypothèse suivante: dans le contexte phonétique, la consonne influerait, non seulement, sur le formant 1 et 2 mais aussi sur le formant nasal (Fn) de la voyelle nasale et elle l'écarte de son propre « hyperespace », bien qu'elle ne bannisse pas l'anti-formant. Pour vérifier notre hypothèse, nous avons analysé les productions phonétiques de 4 étudiants de français de première année de l'université d'Ispahan à l'aide d'un logiciel d'analyse de sons tout en tenant compte des variables acoustiques des formants 1,2 et 3 . Notre corpus est constitué de 2 phrases collectées lors du deuxième semestre de l'année universitaire 2016-2017. Dans les productions phonétiques des apprenants étudiés, l'effet du contexte phonétique donne lieu au phénomène de ratage de cible (undershoot) des voyelles nasales et empêche surtout la constitution $\mathrm{du}$ formant nasal ou du murmure nasal, d'où l'apparition d'un écart de prononciation.

This research focuses on the impact of phonetic context on the formants 1,2 and 3 of the French nasal vowels. The connected speech consists of the succession of oral and nasal vowels influenced by the consonant context. Using the Stevens' and House's model, production undershoot model, this study sought to investigate the effect of phonetic context on the three French nasal vowels. The aim was to check the following hypothesis : in the phonetic context, the consonant would not only affect the formants 1 and 2 but also the nasal formant (nF) of nasal vowel which causes separation from its own "hyperspace", however this would not lead to the disappearance of antiformant. To do so, we analyzed the phonetic productions of 4 first-year French students at the University of Isfahan using Praat software while taking into account the acoustic variables of formants 1, 2 and 3. Our corpus consists of 2 sentences collected during the second semester of 2016-2017 academic year. The results revealed that the effect of phonetic context would lead to 
the undershoot of nasal vowels which prevents the formation of nasal formant or murmur nasal i.e., a gap in pronunciation.

\section{INDEX}

Mots-clés : FLE, voyelles nasales, production « Target-undershoot », formant nasal, écart de prononciation

Keywords : FFL, nasal vowels, production undershoot model, nasal formant, gap in pronunciation

\section{AUTEURS}

MOHAMD HOSSEIN OTROSHI

Université d'Ispahan (University of Isfahan), Iran mh.otroshi@fgn.ui.ac.ir

\section{KAMYAR ABDOLTAJEDINI}

Université d'Ispahan (University of Isfahan), Iran K.Tajedini@fgn.ui.ac.ir

\section{MARZIEH MEHRABI}

Université de Téhéran (University of Tehran), Iran mehrabi.mrz@ut.ac.ir 